International Journal of Modern Physics C

(C) World Scientific Publishing Company

\title{
Entropic Lattice Boltzmann Simulation of the Flow Past Square Cylinder
}

\author{
Santosh Ansumali \\ Department of Materials, Institute of Polymers, Swiss Federal Institute of Technology (ETH) \\ Sonneggstrasse 3, ML J 19, CH-8092 Zurich, Switzerland \\ E-mail: ansumali@mat.ethz.ch \\ Shyam Sunder Chikatamarla \\ Department of Energy Technology, Indian Institute of Technology, \\ Chennai, India \\ Christos Emmanouil Frouzakis \\ Aerothermochemistry und Combustion Systems Laboratory, Swiss Federal Institute of Technology (ETH) \\ Clausiussstrasse 33, CH-8092 Zürich, Switzerland \\ Konstantinos Boulouchos \\ Aerothermochemistry und Combustion Systems Laboratory, Swiss Federal Institute of Technology (ETH) \\ Sonneggstrasse 3, CH-8092 Zürich, Switzerland \\ Received (received date) \\ Revised (revised date)
}

\begin{abstract}
Minimal Boltzmann kinetic models, such as lattice Boltzmann, are often used as an alternative to the discretization of the Navier-Stokes equations for hydrodynamic simulations. Recently, it was argued that modeling sub-grid scale phenomena at the kinetic level might provide an efficient tool for large scale simulations. Indeed, a particular variant of this approach, known as the entropic lattice Boltzmann method (ELBM), has shown that an efficient coarse-grained simulation of decaying turbulence is possible using these approaches. The present work investigates the efficiency of the entropic lattice Boltzmann in describing flows of engineering interest. In order to do so, we have chosen the flow past a square cylinder, which is a simple model of such flows. We will show that ELBM can quantitatively capture the variation of vortex shedding frequency as a function of Reynolds number in the low as well as the high Reynolds number regime, without any need for explicit sub-grid scale modeling. This extends the previous studies for this set-up, where experimental behavior ranging from $R e \sim O(10)$ to $R e \leq 1000$ were predicted by a single simulation algorithm 12345 .

Keywords: Lattice Boltzmann; $H$ theorem; Turbulence Model; Flow Past Square Cylinder
\end{abstract}

\section{Introduction}

Direct numerical simulations of low to moderate Reynolds number turbulent flows in simple geometries, such as channel or infinite domain, have substantially improved the understanding of turbulent flows. However, for fully developed turbulent 
flows of engineering interest, such simulations are far beyond the processing capabilities of even the most powerful computers. Due to the huge difference between the smallest length scale, $l_{k}$, (known as Kolmogorov length) and the largest length scale (typically on the scale of system-size), all length scales cannot be taken into account in any simulations. On the other hand, the convective non-linearity in the Navier-Stokes equations ensures that hydrodynamic equation at all length scales are coupled to each other. Further complications arise due to the non-linearity introduced through the pressure term and the boundary conditions. Thus, writing model equations for large scale turbulence simulations, which can predict the effects of unresolved (small) scales of motion on the resolved (large) ones constitutes a major part of turbulence research.

Many models for turbulence rely on the idea of scale-separation, which assumes that there exist a cut-off length scale, $l_{\mathrm{c}},\left(l_{\mathrm{c}} \gg l_{k}\right)$ such that small-scale eddies, $l<l_{\mathrm{c}}$, are in local equilibrium with large scale eddies $\left(l>l_{\mathrm{c}}\right)$. For example, all eddyviscosity models rely on the analogy between turbulent and molecular transport. Within this analogy, the small-scale eddies play the role of the molecules, while the Kolmogorov length-scale $l_{k}$, (in actual computations replaced by the grid spacing $\delta$ ) plays the role of the mean-free path. This analogy with kinetic theory has the significant advantage of leaving the form of the Navier-Stokes equation for the large eddies intact, with an effective viscosity $\nu_{e}$ replacing the molecular viscosity $\nu$.

However, the scale-separation argument discussed above simply does not hold for turbulence. Thus, these models often fail to reproduce strongly off-equilibrium turbulent effects. In particular, the scenario described by the eddy viscosity model clearly indicates that the dynamics of the smallest resolved scales $\left(l \rightarrow l_{c}\right)$ faces a situation similar to finite-Knudsen flows at $K n \sim 1$. This lack of scale separation is the fundamental reason for the failure to develop a consistent theory of fully developed turbulence. For the classical Boltzmann equation, the problem of $K n \sim 1$ hydrodynamics was solved by the method of invariant manifolds 6778 .

It has been recently pointed out that the kinetic representation of hydrodynamics provides a natural generalization of the notion of eddy viscosity to such non-equilibrium high- $K n_{\mathrm{t}}$ regimes 9101112, $\left(K n_{\mathrm{t}}=l_{k} / l\right.$, for an eddy of size $\left.l\right)$. The key point is that solutions of the kinetic equation apply at all orders of the Knudsen number, so that any kinetic model ensuring correct hydrodynamic behavior would handle the dynamics of small eddies at $K n_{t} \sim 1$ in a way which goes beyond the eddy viscosity representation.

Further, important computational features of the kinetic models are the linearity of the streaming operator and the locality (in configuration space) of the the non-linearity introduced by the collision term. In order to efficiently use these advantages of kinetic models, while getting rid of enormously large numbers of degree of freedoms associated with the (true) Boltzmann equation, a drastically simplified versions of the Boltzmann equation, known as the lattice Boltzmann method 1314 (hereafter LBM), had been developed in the last decade. Indeed, the method has been applied to a large variety of fluid flows, including turbulent ones 1215]16. 
However, owing to the lack of a H-theorem 17, the standard lattice Boltzmann method often exhibits disruptive non-linear instabilities associated with the dynamics of near-grid scales with $l \sim \delta$. In order to improve on this shortcoming of the method, while preserving its computational efficiency, a modification of the algorithm, known as "entropic lattice Boltzmann method", was developed recently 1820212325 . The compliance with the $H$-theorem ensures the non-linear stability for the method. The basic idea is to exploit the requirement of increase in the entropy ("grid entropy") as a criterion to decouple the resolved scales from the unresolved ones. The compliance with the $\mathrm{H}$-theorem ensures that the termination of the cascade picture on the grid scale does not lead to non-physical disruptive non-linear instabilities.

In fact, the model with its adaptive but local regulation of the relaxation time, can be regarded as a turbulence model inspired by genuinely kinetic requirements. The efficiency of such turbulence modeling was recently tested in the context of the decaying turbulence 24. In this work, we test the efficiency of the method for the flow past a square cylinder, a prototype of flows of engineering interest. This set-up is used often to test the efficiency of different Navier-Stokes (or its model) solvers (for example see Refs. 1-5). Even though the set-up is simple enough, experimental results predict a non-trivial dependence of the vortex-shedding frequency on the Reynolds number 26. The vortex-shedding frequency is characterized by a dimensionless number known as Strouhal number, which is defined as $S=n L_{\text {char }} / U_{0}$, where $n$ is the frequency of the vortex shedding, $L_{\text {char }}$ is the length of the cylinder, and $U_{0}$ is the characteristic velocity, taken as the velocity at the inlet. The Strouhal number undergoes a sharp transition around $R e \sim 70$ and attends a more or less constant value of 0.13 at high Reynolds numbers 2627. Although the transition at $R e \sim 70$ is captured by different schemes with varying degree of accuracy, so far to the best of our knowledge using direct Numerical simulation of Navier-Stokes equation, the experimental curve is reproduced up to $R e=1000$. Various models of turbulence have also been tested on the flow past a square cylinder at $R e=21400$ (28)29303132333435). We will show that ELBM can quantitatively capture the variation of vortex shedding frequency as a function of Reynolds number without any need for explicit sub-grid scale modeling.

The work is organized as follows: A brief description of the LBM and its entropic version is provided in Sec. II. In Sec. III, implementation details for the flow past a square cylinder will be provided. In Sec. IV, a comparison of the present work with the experimental results and other numerical results will be presented. Further, in Sec. V, the outlook for the present work will be discussed.

\section{The entropic Lattice Boltzmann method}

In the lattice Boltzmann method, the discrete kinetic equation for the one particle distribution function, $f_{i} \equiv f\left(x, \mathbf{C}_{i}, t\right)$, which denotes the probability of finding a 
particle with velocity $\mathbf{C}_{i}$ at position $x$ and time $t$, is written as:

$$
\partial_{t} f_{i}+\mathbf{C}_{i} \cdot \partial_{\mathbf{x}} f_{i}=-\tau^{-1}\left(f_{i}-f_{i}^{\text {eq }}\right),
$$

where, the right-hand-side of this equation represents collisional relaxation to the local equilibrium, $f_{i}^{\text {eq }}$, on a time scale $\tau$. The irreversible relaxation to this local equilibrium provides viscous behavior, with a kinematic viscosity of the order of $\nu \sim c_{s}^{2} \tau$, where $c_{s}$ is the speed of sound. A general formulation of the kinetic models is presented in the work of Gorban and Karlin 36 .

The set of discrete velocities is chosen in such a way as to ensure sufficient symmetry to recover the conservation of mass, momentum and momentum-flux. The minimal set of discrete velocities needed to reconstruct the Navier-Stokes equations (as the large-scale limit) are related to zeroes of third-order Hermite polynomials in $\mathbf{C}_{i}$. For example in one-dimension (spatial dimension $D=1$ ), the three discrete velocities are 37:

$$
\mathbf{C}=c\{-1,0,1\},
$$

where $c$ is some arbitrary constant usually taken as $c=\delta x / \delta t$, with $\delta t$ and $\delta x$ being step-size in the spatial and the temporal discretization, respectively. In higher dimensions, the discrete velocities are tensor products of the discrete velocities in one dimension.

The discrete-velocity local equilibrium is the minimizer of the the discrete $H$ function:

$$
H_{\left\{W_{i}, \mathbf{C}_{i}\right\}}=\sum_{i=1}^{3^{D}} f_{i} \ln \left(\frac{f_{i}}{W_{i}}\right)
$$

where, $W_{i}$ are the weights associated with each discrete velocity (for the onedimensional case)

$$
W=\left\{\frac{1}{6}, \frac{2}{3}, \frac{1}{6}\right\},
$$

under the constraint of local conservation laws:

$$
\sum_{i=1}^{3^{D}} f_{i}^{\mathrm{eq}}\left\{1, \mathbf{C}_{i}\right\}=\{\rho, \rho \mathbf{u}\}
$$

In higher dimensions, the weights are constructed by multiplying weights associated with each direction.

The explicit expression for $f_{i}^{\text {eq }}$ is 23 .

$$
f_{i}^{\mathrm{eq}}=\rho W_{i} \prod_{j=1}^{D}\left(2-\sqrt{1+3 u_{j}^{2}}\right)\left(\frac{2 u_{j}+\sqrt{1+3 u_{j}^{2}}}{1-u_{j}}\right)^{c_{i j} / c},
$$

with $j$ being the index for spatial directions. 
In order to discretise the model kinetic equation in a way consistent with the second law of thermodynamics ( $H$-theorem), the notion of the bare collision $\boldsymbol{\Delta}$, defined as the collision term stripped of its relaxation parameters, is introduced. For the case of the single relaxation time collision model considered here (right hand side of Eq. 1), the bare collision is given as $\boldsymbol{\Delta}=\mathbf{f}_{\text {eq }}-\mathbf{f}$, where $\mathbf{f}$ denotes the $3^{D}$-dimensional population vector. The time stepping in this method is performed through an over-relaxation collisional process and linear convection through a sequence of steps in which the $H$ function is bound not to decrease. The monotonicity constraint on the $H$ function is imposed through the following geometric procedure: In the first step, populations are changed in the direction of the bare collision in such a way that the $H$ function remains constant. In the second step, dissipation is introduced and the magnitude of the $H$ function decreases. Thus,

$$
f_{i}(\mathbf{x}, \delta t)=f_{i}\left(\mathbf{x}-\mathbf{C}_{i} \delta t, 0\right)+\alpha \beta\left[f_{i}^{\mathrm{eq}}\left(\mathbf{x}-\mathbf{C}_{i} \delta t, 0\right)-\mathbf{f}\left(\mathbf{x}-\mathbf{C}_{i} \delta t, 0\right)\right]
$$

were $\beta$ is the discrete form of the relaxation frequency related to $\tau$ as

$$
\beta=\frac{\delta t}{2 \tau+\delta t}
$$

and the parameter $\alpha$ is defined by the condition:

$$
H(\mathbf{f})=H(\mathbf{f}+\alpha \boldsymbol{\Delta}) .
$$

Close to the local equilibrium $\alpha$ is equal to 2 (Implementation details can be found in Ref. 20 ). Equation 9 is the essence of the discrete time $H$-theorem for the discrete kinetic equation [7 The notion of the discrete time $H$ theorem was first introduced by Karlin et al 38 . It is the extension of the earlier general study of the initial layer problem in dissipative kinetics 39 40. The local adjustments of the relaxation time (via the parameter $\alpha$ ), as dictated by compliance with the $H$ theorem, guarantee positivity of the distribution function also for the case of discrete steps, thereby ensuring non-linear stability of the numerical scheme.

In this method, for the solid-fluid boundary condition a discretization scheme of diffusive boundary condition for the Boltzmann equation is used 22. The essence of the diffusive boundary condition is that particles loose their memory of the incoming direction after reaching the wall. Once a particle reaches the wall, it gets redistributed in a way consistent with the mass-balance and normal-flux conditions. Further, the boundary condition must also satisfy the condition of detailed balance: if the incoming populations are at equilibrium (corresponding to the wallvelocity), the outgoing populations are also at equilibrium (corresponding to the wall-velocity). As an example, let us consider the case when the wall normal, e, (pointing towards the fluid) is in the positive $y$ direction (see Fig. 1). For this particular case, the boundary update rules for incoming and grazing populations 


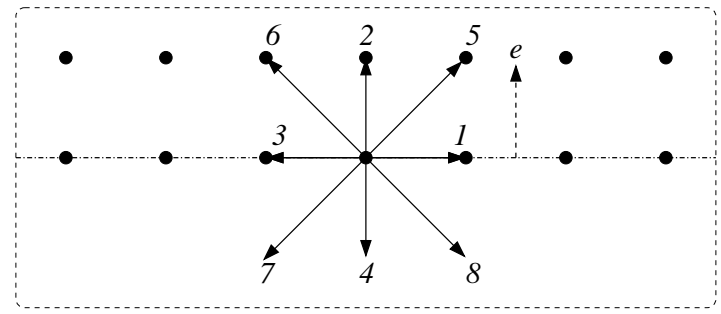

Figure 1: A schematic diagram for situation near a flat wall, when the wall normal, e, (pointing towards the fluid) is in the positive $y$ direction. Here, the case of two-dimensional D2Q9 lattice is considered.

on a two-dimensional lattice are:

$$
\begin{aligned}
f_{0}(x, y, t+\delta t) & =f_{0}^{*}(x, y, t), \\
f_{1}(x, y, t+\delta t) & =f_{0}^{*}(x-c \delta t, y, t), \\
f_{2}(x, y, t+\delta t) & =f_{0}^{*}(x+c \delta t, y, t), \\
f_{4}(x, y, t+\delta t) & =\frac{1}{2}\left[f_{4}^{*}(x, y+c \delta t, t)+f_{4}^{*}(x, y, t)\right], \\
f_{7}(x, y, t+\delta t) & =\frac{1}{2}\left[f_{7}^{*}(x+c \delta t, y+c \delta t, t)+f_{7}^{*}(x, y, t)\right], \\
f_{8}(x, y, t+\delta t) & =\frac{1}{2}\left[f_{8}^{*}(x-c \delta t, y+c \delta t, t)+f_{8}^{*}(x, y, t)\right],
\end{aligned}
$$

where $f^{*}$ denotes after collision populations, and update rules for outgoing populations are:

$$
\begin{aligned}
& f_{2}(\mathbf{x}, t+\delta t)=f_{2}^{\mathrm{eq}}\left(\rho, \mathbf{u}_{\mathrm{wall}}\right) \frac{f_{4}(\mathbf{x}, t+\delta t)+f_{7}(\mathbf{x}, t+\delta t)+f_{8}(\mathbf{x}, t+\delta t)}{f_{2}^{\mathrm{eq}}\left(\rho, \mathbf{u}_{\text {wall }}\right)+f_{5}^{\mathrm{eq}}\left(\rho, \mathbf{u}_{\text {wall }}\right)+f_{6}^{\mathrm{eq}}\left(\rho, \mathbf{u}_{\text {wall }}\right)} \\
& f_{5}(\mathbf{x}, t+\delta t)=f_{5}^{\mathrm{eq}}\left(\rho, \mathbf{u}_{\text {wall }}\right) \frac{f_{4}(\mathbf{x}, t+\delta t)+f_{7}(\mathbf{x}, t+\delta t)+f_{8}(\mathbf{x}, t+\delta t)}{f_{2}^{\mathrm{eq}}\left(\rho, \mathbf{u}_{\text {wall }}\right)+f_{5}^{\mathrm{eq}}\left(\rho, \mathbf{u}_{\text {wall }}\right)+f_{6}^{\mathrm{eq}}\left(\rho, \mathbf{u}_{\text {wall }}\right)} \\
& f_{6}(\mathbf{x}, t+\delta t)=f_{6}^{\mathrm{eq}}\left(\rho, \mathbf{u}_{\text {wall }}\right) \frac{f_{4}(\mathbf{x}, t+\delta t)+f_{7}(\mathbf{x}, t+\delta t)+f_{8}(\mathbf{x}, t+\delta t)}{f_{2}^{\mathrm{eq}}\left(\rho, \mathbf{u}_{\text {wall }}\right)+f_{5}^{\mathrm{eq}}\left(\rho, \mathbf{u}_{\text {wall }}\right)+f_{6}^{\mathrm{eq}}\left(\rho, \mathbf{u}_{\text {wall }}\right)}
\end{aligned}
$$

\section{Implementation Details for Flow Past a Square Cylinder}

We study the flow past a square cylinder in the two-dimensional set-up shown schematically in Fig. 22 The square cylinder is placed at a location $L_{1}=10 L_{\text {char }}$ downstream form the inlet and symmetric in the other direction. For high Reynolds number simulations (above $R e \geq 1000$ ), a computational grid of width $W=25 L_{\text {char }}$ and length $L=45 L_{\text {char }}$ was used, while for moderate Reynolds number simulations (above $R e<1000$ ), a computational grid of width $W=25 L_{\text {char }}$ and length $L=$ $30 L_{\text {char }}$ was used. The width of the domain results in a blockage ratio $\left(L_{\text {char }} / W\right)$ of less than 5. In all simulations the inlet velocity $U_{0}$ is specified as $U_{0}=0.05$ (in lattice units). 


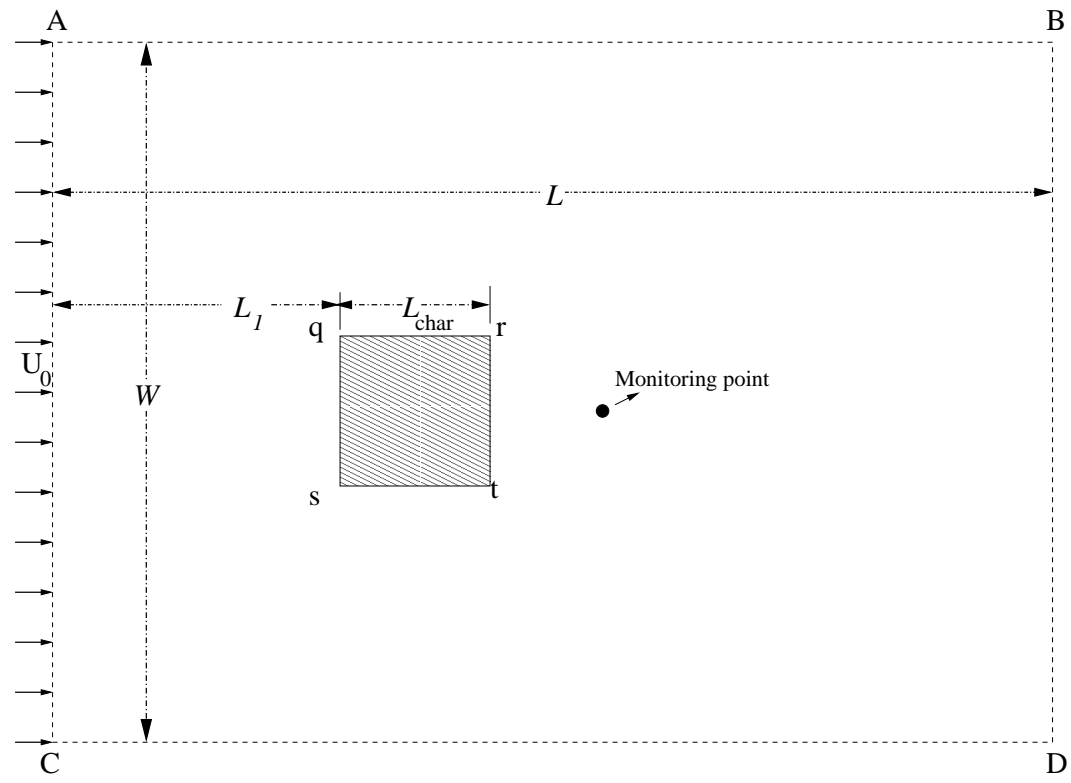

Figure 2: Setup for the simulation of flow past square obstacle

At the inlet (side AC in Fig. 2), the populations are replaced with the equilibrium values that correspond to the inlet velocity and density. As, the simulation result is not very sensitive to the exact condition specified at the inlet this simple lowest order approximation at the inlet is sufficient. However, the simulation is very sensitive to the condition employed at the exit (side BD in Fig. 2), which should be provided in a way that does not affect the bulk flow strongly. The sensitivity of the outlet condition is known for this problem 113. We use the simplest possible strategy of taking a domain long enough in the direction of flow (up to $45 L_{\text {char }}$ ). At the exit, the populations pointing towards the domain are simply replaced by the equilibrium values that correspond to the extrapolated velocity and density. On the top and bottom surfaces (side AB and CD in Fig. 2), the free-slip boundary condition is imposed 13 . On the cylinder walls (square qrst in Fig. 2), the diffusive boundary condition described in the previous section is used.

The analysis of the unsteady data is performed using the discrete Fourier transform. The vortex shedding frequency, $n$, is obtained from the discrete Fourier transform (in time) of the instantaneous velocity at a monitoring point. The unsteady data is collected from two or three point downstream of the square cylinder.

\section{A comparison of Experiment and Simulation}

Numerical simulations of the unsteady flow in the wake of rectangular cylinders immersed in an infinite stream have been carried out by many authors in the past. Much of the effort concentrated either in studying the behavior for moderate 


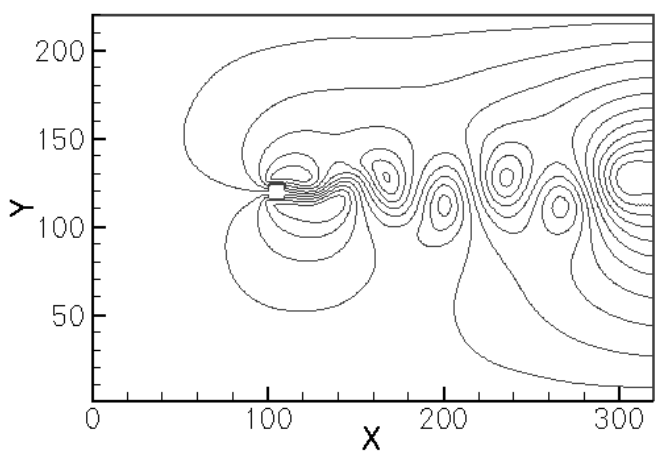

Figure 3: A snap-shot of streamfunction at $R e=100$ with $L_{\text {char }}=10$.

Reynolds number flows 261234 or verifying sub-grid models of turbulence using experimental data at high Reynolds number2829|30|31|32|333435] (Re=21400).

In this work, we will investigate the behavior of Strouhal number as a function of Reynolds number for moderate as well as high Reynolds number flows. Experimentally, two distinct regimes are known for this flow 26]. First, a sharp transition in the value of the Strouhal number is observed for Reynolds number, $R e \sim 70$. Further, for high Reynolds number flows (Re $\gg 1000)$, the Strouhal number is almost independence of the Reynolds number and attainds an almost constant value of 0.13. We will show that indeed using entropic lattice Boltzmann method the experimental behavior can be predicted in a quantitative fashion.

In Figures 3 and 4 show the streamlines for $R e=100$ and $R e=3000$, respectively, after the initial transient dies out. As it can be seen from the plot, at high Reynolds number the numerical simulation is not well resolved. In addition, the errors from the outflow boundary starts to corrupt the simulation.

Fig. [5 shows a comparison of the experimental 26 and simulation results. Table 11 summarizes our simulation results with some of the earlier reported works. It is clear that the present method is able to capture the transition around $R e \sim 70$ as well as the asymptotic behavior at high Reynolds numbers in a quantitatively correct fashion. It is worth mentioning that the resolution used for the high Reynolds number simulations $(R e \gg 250)$ is quite low in comparison to the standard lattice Boltzmann method. In order to numerically simulate this flow for $R e \gg 1000$, using a standard lattice Boltzmann method the required number of grid-points would be at least $O\left(10^{7}\right)$ to avoid numerical instabilities. The fact that an underresolved simulation (number of grid-points used is $O\left(10^{5}\right)$ ), without any explicit sub-grid scale 


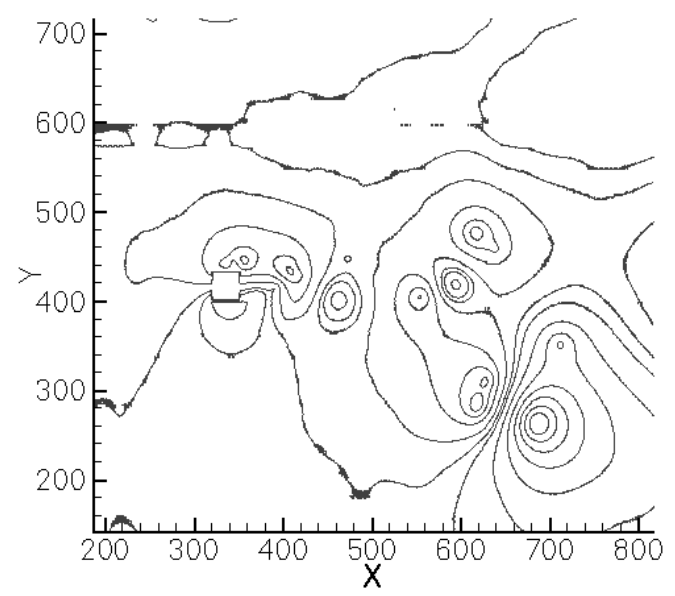

Figure 4: A snap-shot of streamfunction at $R e=3000$ with $L_{\text {char }}=24$.

model, can predict the experimentally observed variation of the Strouhal number (a coarse-feature of the flow) indicates that the present method is a candidate for efficient coarse-grained simulations of high Reynolds numbers flows. Similar results were obtained in the case of homogeneous decaying turbulence 24 .

Finally, at very-high Reynolds number $R e=20000$, with a grid of $L_{c h a r}=32$ and $L=45 L_{\text {char }}$ the Strouhal number is fluctuating between $0.09-0.15$, and errors from the boundary corrupted the simulation before the Strouhal number can reach a final steady state.

Table 1. Variation of Strouhal number as a function of Re.

\begin{tabular}{c|c|c|c|c|c|} 
Re & Experiment 26 & ELBM & LBM & FD 1 & FV2 \\
\hline 37 & N. A. & 0.098 & N. A. & N. A. & N. A. \\
81 & $0.115-0.130$ & 0.122 & 0.132 & - & 0.132 \\
110 & $0.143-0.145$ & 0.141 & 0.15 & - & N. A. \\
250 & $0.140-0.143$ & 0.137 & - & $0.16-0.17$ & 0.154 \\
500 & $0.125-0.127$ & - & - & - & 0.174 \\
1000 & $0.122-0.123$ & 0.123 & - & $0.14-0.15$ & N. A. \\
2800 & $0.128-0.129$ & - & N.A & $0.14-0.15$ & N. A. \\
3000 & $0.128-0.129$ & 0.133 & N.A & N.A. & N. A. \\
5000 & $0.127-0.131$ & 0.134 & N.A & N.A. & N. A. \\
20000 & $0.13-0.134$ & $0.09-0.15$ & N.A & N.A. & N. A.
\end{tabular}

Before concluding we will like to comment on the importance of the dimensionality of the flow. Above some critical Reynolds number, the flow becomes threedimensional. Nevertheless, our two-dimensional simulations accurately capture the Strouhal number dependence on the Reynolds number. We are currently extending 


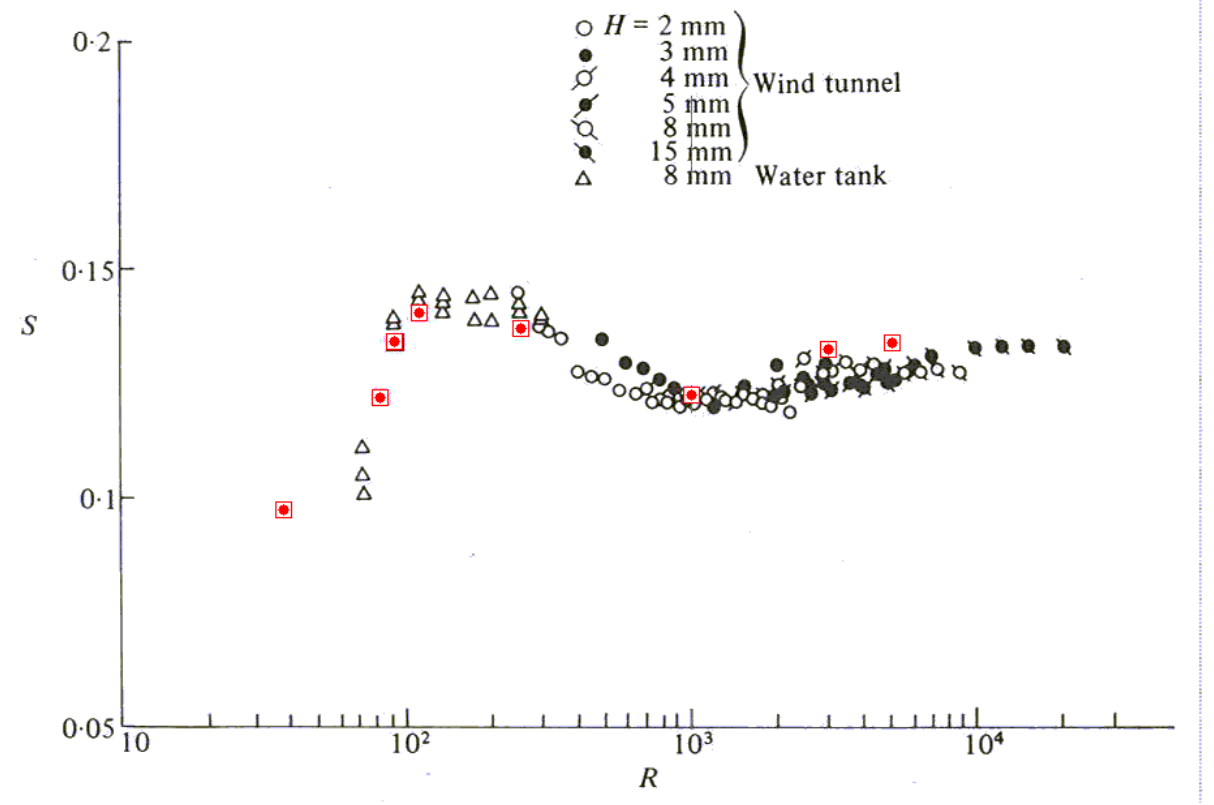

Figure 5: A comparison of the present simulation with the available experimental result (Ref. 26). The figure shows the variation of the Strouhal number as a function of Reynolds number. The simulation points are shown as rectangles with a filled circle. 
the code to study the three-dimensional effects in detail.

\section{Conclusions}

We have shown that ELBM can quantitatively predict the variation of vortex shedding frequency as a function of Reynolds number even for $R e>>1000$ for the flow past a square cylinder. This shows that at least large scale features of the motion are effectively captured by the built-in sub-grid scale model of the ELBM. Further detailed study using three-dimensional model are needed to check the accuracy and efficiency of the method for $R e \sim O(10000)$.

\section{Acknowledgments}

We acknowledge Dr. Iliya V. Karlin for suggesting the statement of the problem and guidance, Prof. A. Gorban for discussions on the boundary conditions, Prof. V. Babu for providing the postprocessing subroutines, Prof. H. C. Öttinger, and Prof. S. Succi for useful discussions.

1. R. W. Davis, and E. F. Moore, J. Fluid Mech. 116,p. 475 (1982).

2. A. Sohankar, C. Norberg, and L. Davidson, Phys. Fluids 11, p. 288 (1999).

3. M. Bruer, J. Bernsdorf, T. Zeiser and D. Durst, Int. J. Heat Fluid Flow 21, p. 186 (2000).

4. A. Lamura, G. M. Gompper, T. Ihle, D. M. Kroll, Europhys Lett. 56, p. 319 (2001).

5. A. Lamura, G. M. Gompper, Europhys J. E 9, p. 477 (2002).

6. A. N. Gorban, and I.V. Karlin, Advance Modeling and Analysis C 33, p. 39 (1992).

7. A. N. Gorban, and I.V. Karlin, Transport Theory Statist. Phys. 23, p. 559 (1994).

8. I.V. Karlin, J. Phys. A 33, p. 8037 (2000).

9. H. Chen, S. Succi, and S. Orszag, Phys. Rev. E 59,p. R2527 (1999).

10. S. Succi, O. Filipova, H. Chen, and S. Orszag, J. Stat. Phys. 107,p. 261 (2002).

11. S. Succi, I. V. Karlin, H. Chen, and S. Orszag, Physica A 280,p. 92 (2000).

12. H. Chen, S. Kandasamy, S. Orszag, R. Shock, and S. Succi, Science 301,p. 633 (2003).

13. S. Succi, The Lattice Boltzmann Equation for Fluid Dynamics and Beyond (Oxford University Press, Oxford, 2001 ).

14. R. Benzi, S. Succi and M. Vergassola, Phys. Rep. 222, p. 145 (1992).

15. R. Benzi, S. Succi, J. Phys. A 23, p. L1 (1990).

16. D. O. Martinez, W. H. Matthaeus, and S. Chen, and D. C. R. Montgomery, Phys. Fluids 6, p. 1285 (1994).

17. S. Succi, I. V. Karlin, and H. Chen, Rev. Mod. Phys. 74,p. 1203 (2002).

18. I. V. Karlin, A. Ferrante and H. C. Öttinger, Europhys. Lett. 47, p. 182 (1999).

19. S. Ansumali and I. V. Karlin, Phys. Rev. E 62, 7999 (2000).

20. S. Ansumali and I. V. Karlin, J. Stat. Phys. 107, 291 (2002).

21. S. Ansumali and I. V. Karlin, Phys. Rev. E 65, 056312 (2002).

22. S. Ansumali and I. V. Karlin, Phys. Rev. E 66, 026311 (2002).

23. S. Ansumali, I. V. Karlin and H. C. Öttinger, Europhys. Lett. 63, 798 (2003).

24. I.V. Karlin, S. Ansumali, E.D. Angelis, H. C. Öttinger, and S. Succi, arXiv:cond-mat/0306003 (2003).

25. B. M. Boghosian, J. Yepez, P. V. Coveney, and A. J. Wagner, Proc. Roy. Soc. Lond. A 457, p. 717 (2001).

26. A. Okajima, J. Fluid Mech. 116, p. 379 (1982). 
27. B. J. Vickery, J. Fluid Mech. 25, p. 481 (1966).

28. G. Bosch, and W. Rodi, Int. J. Numer. Meth. Fluids 28, p. 601 (1998).

29. P. R. Voke, in ERCOFTAC Series: Direct and Large Eddy Simulation II, ed. J. P. Chollet, P. R. Voke, and L. Kleiser p. 355 (1997).

30. M. Pourquie, M. Breuer, and W. P. Rodi, in ERCOFTAC Series: Direct and Large Eddy Simulation II, ed. J. P. Chollet, P. R. Voke, and L. Kleiser p. 375 (1997).

31. R.W.C.P. Verstappen, and A. Veldman, in ERCOFTAC Series: Direct and Large Eddy Simulation II, ed. J. P. Chollet, P. R. Voke, and L. Kleiser p. 381 (1997).

32. S. Murikami, S. Iizuka, A. Mochida, and Y. Tominaga, in ERCOFTAC Series: Direct and Large Eddy Simulation II, ed. J. P. Chollet, P. R. Voke, and L. Kleiser p. 385 (1997).

33. G. Wang, and S. P. Vanka, in ERCOFTAC Series: Direct and Large Eddy Simulation II, ed. J. P. Chollet, P. R. Voke, and L. Kleiser p. 397 (1997).

34. T. Kogaki and T. Kobayashi and N. Taniguchi, in ERCOFTAC Series: Direct and Large Eddy Simulation II, ed. J. P. Chollet, P. R. Voke, and L. Kleiser p. 401 (1997).

35. K. Nozawa, and T. Tamura, in ERCOFTAC Series: Direct and Large Eddy Simulation II, ed. J. P. Chollet, P. R. Voke, and L. Kleiser p. 409 (1997).

36. A. N. Gorban, and I.V. Karlin, Physica A 206, p. 401 (1994).

37. X. Shan, and X. He, Phys. Rev. Lett. 80, p. 65 (1998).

38. I. V. Karlin, A. N. Gorban, S. Succi and V. Boffi, Phys. Rev. Lett. 81, 6 (1998).

39. A. N. Gorban, I. V. Karlin, V. B. Zmievskii and T. F. Nonnenmacher, Physica A 231, 648 (1996).

40. A. N. Gorban, I. V. Karlin and V. B. Zmievskii, Transport Theory and Stat. Phys. 28, 271 (1999). 